\title{
Médiévales
}

Langues, Textes, Histoire

47 | automne 2004

Îles du Moyen Âge

\section{Récits d'îles. Espace insulaire et poétique du récit dans l'Estoire del saint Graal}

Tales of the Isles. Insular Setting and the Poetics of Narrative in the

\section{Mireille Séguy}

\section{OpenEdition}

1 Journals

\section{Édition électronique}

URL : https://journals.openedition.org/medievales/504

DOI : 10.4000/medievales.504

ISSN : $1777-5892$

\section{Éditeur}

Presses universitaires de Vincennes

\section{Édition imprimée}

Date de publication : 1 décembre 2004

Pagination : 79-96

ISBN : 2-84292-157-7

ISSN : 0751-2708

\section{Référence électronique}

Mireille Séguy, «Récits d'îles. Espace insulaire et poétique du récit dans l'Estoire del saint Graal », Médiévales [En ligne], 47 | automne 2004, mis en ligne le 02 septembre 2006, consulté le 23 avril 2022. URL : http://journals.openedition.org/medievales/504; DOI : https://doi.org/10.4000/medievales.504

Ce document a été généré automatiquement le 23 avril 2022.

Tous droits réservés 


\title{
Récits d'îles. Espace insulaire et poétique du récit dans l'Estoire del saint Graal
}

Tales of the Isles. Insular Setting and the Poetics of Narrative in the

\author{
Mireille Séguy
}

L'Estoire del saint Graal constitue le premier volet du plus grand cycle romanesque en prose composé au XIII ${ }^{e}$ siècle (entre 1215 et 1235 environ), le Lancelot-Graal. Ce roman privilégie très largement l'espace insulaire : plus du tiers de l'histoire racontée se passe en séjours dans des îles, ou en navigations d'île en île. Bien entendu, l'Estoire del saint Graal n'est ni le seul ni le premier roman de la matière de Bretagne à comprendre des navigations ponctuées par des séjours insulaires, sur le modèle de la Navigation de saint Brendan ${ }^{1}$. Il se trouve cependant qu'aucun autre roman arthurien n'accorde autant d'importance que l'Estoire à l'île, moins comme thème narratif que comme espace diégétique privilégié et surtout, nous aimerions le montrer, comme espace dans et par lequel le récit trouve à résoudre les principaux problèmes que soulève son élaboration. Que la mise en œuvre du récit de l'Estoire soit problématique tient essentiellement à la fonction et à la situation singulières que cette oeuvre assume au sein du Lancelot-Graal. Roman inaugural du cycle, l'Estoire a essentiellement pour charge de revenir sur les origines du Graal succinctement fixées par le Joseph de Robert de Boron et surtout de rendre compte des moyens par lesquels la relique a été transportée par Joseph d'Arimathie et son fils Josephé d'Orient en Occident, c'est-à-dire de Palestine en Grande-Bretagne - laquelle n'est pas encore le royaume d'Arthur (il s'en faut à peu près de 350 ans). Tout en s'inscrivant comme un récit des commencements, l'Estoire doit donc également couvrir de l'espace, créer des itinéraires, trouver des liens entre l'immense fonds culturel de l'Antiquité païenne et une fiction arthurienne dont la christianisation est achevée. Déjà ambitieuse en soi, l'entreprise de l'Estoire est compliquée du fait que ce récit a très vraisemblablement été composé après l'ensemble du cycle dont il doit fixer les commencements et assurer la cohésion ${ }^{2}$. Le statut fondateur du roman n'étant confirmé par aucun des récits qui le suivent, il lui revient 
de forger lui-même les indices d'antécédence susceptibles de l'imposer, a posteriori, comme le récit d'origine du cycle. Les lignes qui suivent tentent de tester l'hypothèse selon laquelle l'espace insulaire, tel qu'il est configuré par l'écriture romanesque, offre, parallèlement à d'autres dispositifs que nous n'envisagerons pas ici, des solutions pour résoudre la double difficulté à laquelle l'Estoire se trouve confrontée. La première est inhérente à la complexité du projet qu'elle poursuit : inscrire les commencements du Graal dans l'immense espace-temps pré-arthurien tout en leur donnant une tonalité chrétienne. La seconde tient à sa situation paradoxale au sein du cycle, situation qui l'oblige à s'inscrire, dans l'après-coup, comme le récit fondateur d'un très vaste ensemble constitué de massifs narratifs hétérogènes ${ }^{3}$.

Un espace paradoxal

2 Durant toute la seconde partie du récit et une partie de la troisième, les personnages principaux avancent dans l'espace essentiellement au gré de navigations (ou de transports divins, mais cela revient la plupart du temps au même) qui les conduisent dans différentes îles où ils peuvent, à l'occasion, se rencontrer. Un résumé de ces différents déplacements, même schématique, donnera une idée suffisante de leur complexité. Alors qu'il se trouve dans la capitale de son royaume, la ville de Sarras, le roi Mordrain (un roi païen dont la première partie du récit raconte les aventures et la conversion) est transporté par le saint Esprit dans l'île de Port Péril. Après y avoir subi un certain nombre d'épreuves, il quitte cette île en embarquant sur un grand navire qui l'emporte vers une destination inconnue. Peu après, Nascien, son beau-frère, est déposé par la volonté divine sur l'île Tournoyante, où son fils Célidoine accoste un peu plus tard, à bord de la Nef de Salomon - navire qu'il a échangé, en pleine mer, contre son embarcation première, une nef où il voyageait en compagnie d'un lion. Ils repartent tous deux dans la Nef de Salomon, où se trouve, entre autres objets symboliques, l'épée du roi David. Ils accostent ensuite dans une île qui n'est pas nommée dans le texte, et que l'on pourra appeler ici l'île de l'épée brisée, puisque Nascien y brise l'épée du roi David en combattant contre un géant. Après avoir vaincu le géant, les personnages quittent l'île, toujours à bord de la Nef de Salomon, retrouvent en mer Mordrain, puis repartent tous dans la vaste embarcation de celui-ci. Entre-temps, la reine Flegetine, épouse de Nascien, a envoyé des messagers à la recherche de son mari. Ceux-ci ne tardent pas à faire naufrage dans une quatrième île, l'île d'Hippocrate, d'où ils repartent le surlendemain à bord de la nef au lion (première embarcation de Célidoine) qu'un mystérieux vieil homme leur cède fort opportunément. Trois jours plus tard, ils retrouvent en mer Nascien, Célidoine et Mordrain qu'ils rejoignent dans leur nef. Tous finissent par gagner le royaume de Sarras. La nuit de leur arrivée, Joseph d'Arimathie, son fils Josephé et leurs compagnons parviennent de leur côté en Grande-Bretagne ${ }^{4}$. Il faudra plusieurs mois de navigation à Nascien pour les y rejoindre. Quant à Mordrain, il fera la traversée un peu plus tard, dans le but de secourir ses compagnons emprisonnés par le roi païen Crudel. Alors que l'évangélisation de la Grande-Bretagne par Joseph et Josephé se poursuit, une cinquième île, l'île du roi Orcauz, constitue le cadre des aventures du personnage de Pierre, un parent de Joseph d'Arimathie.

3 Ces parcours croisés, puis convergents et enfin successifs suggèrent essentiellement deux remarques :

4 - La première a trait à la nature singulière de ces itinéraires. Ils se laissent en effet moins appréhender en termes de tracés qu'en termes d'intervalles parcourus, entre les continents et les îles d'abord, mais aussi et surtout entre les îles elles-mêmes - pour ne 
pas parler des trajets effectués entre les différentes embarcations, qui peuvent faire figure d'espaces insulaires mouvants où les personnages peuvent se rencontrer, subir des aventures, rejoindre d'autres embarcations, etc. Si l'on considère que l'Estoire assimile explicitement la mer au monde, selon une métaphore topique dans la tradition exégétique chrétienne ${ }^{5}$, on peut lire ces itinéraires d'île en île, itinéraires fragmentés qui dessinent un espace en pointillés, non seulement comme une manière d'inscrire de l'espace dans le récit mais aussi comme une manière de représenter l'espace dans le récit. À suivre en particulier les analyses de Paul Zumthor, l'espace médiéval s'appréhende essentiellement, en effet, comme un intervalle entre deux points: « l'espace médiéval est [...] ce qui est entre deux : un vide à remplir. On ne le fait exister qu'en le parsemant de sites $»^{6}$.

5 - La seconde remarque, sans doute celle qui s'impose le plus immédiatement, tient à la nature paradoxale de l'espace gagné tout au long de cette seconde partie. S'il est indubitable que les personnages y progressent, cette progression, pour une large part erratique, est à la fin annulée (puisqu'ils se retrouvent tous au terme de leur périple à leur point de départ). Le déplacement spatial majeur du récit, c'est-à-dire le passage d'Orient en Occident, s'effectue quant à lui plus tard, et n'occupe, suivant les personnages, que quelques lignes ou quelques paragraphes. La conclusion qui s'impose est que l'espace qui se gagne à voyager d'île en île dans l'Estoire n'est pas un espace géographique. Le texte se situe ici dans la droite ligne de la Navigation de saint Brendan, pour ne prendre qu'une référence en langue vernaculaire, où l'île marque les étapes d'une avancée plus spirituelle que topographique, plus symboliquement que référentiellement marquée ${ }^{7}$.

Que l'île soit dans l'Estoire essentiellement un lieu du non-lieu, un lieu où se perdent tous les repères géographiques, est de fait affirmé systématiquement dans le récit: les cinq îles où séjournent les personnages sont des îles « etranges " (l'adjectif est utilisé explicitement dans trois $\operatorname{cas}^{8}$ ), à la lettre étrangères, hors du monde objectif. Les personnages y abordent invariablement sans savoir comment, qu'ils y soient transportés directement par l'opération du saint Esprit, ou bien conduits au terme d'une navigation dont ils ne maitrisent rien. Ni le thème de la navigation apparemment erratique d'île en île, ni le caractère difficilement situable du séjour insulaire ne sont bien entendu des thèmes neufs; ils sont tous deux, comme on le sait, des éléments majeurs de l'Odyssée comme des récits de navigation celtiques (immrama), éléments abondamment repris et réadaptés par les textes hagiographiques chrétiens. La singularité de l'Estoire est d'insister non pas tant sur l'éloignement ou l'isolement des îles dont elle parle, ou même sur la difficulté de les localiser précisément, que sur le brouillage que ces îles font subir aux coordonnées de l'espace objectif, c'est-à-dire extralittéraire. Plus que difficilement situables, les îles de l'Estoire instaurent du trouble dans les repères géographiques courants que le récit reprend par ailleurs en charge. Qu'on en juge d'après ces exemples :

7 L'île de Port Péril, dans laquelle Mordrain est emporté par la volonté de Dieu, est d'abord soigneusement située par rapport aux quatre points cardinaux :

Chele roche, si est assise en la mer Occeane, en ichele partie ou li drois trespas est a aller de la terre de Babiloine en la terre d'Escoche et d'Islande et es autres parties d'occident. En che trespas est chele roche et si est de si tres grant hauteche ke on en peut sourveoir toute la mer d'Occident jusc'a ichés lieus ou terre puet estre trovee, et de l'autre part, a destre si comme devers galerne, en peut on veoir la terre de Cordres et toute la fin d'Espaigne. (p. 187, § 304) 
Si l'on suit ces informations, l'île se situe à l'emplacement exact du détroit de Gibraltar, décrit ici comme le lieu de passage («trespas ») par excellence entre Orient et Occident. A cette situation géographique de carrefour et de surplomb vient cependant se superposer une première description topographique qui insiste, à l'inverse, sur le caractère sauvage et isolé de l'île :

La roche [...] si est en tout le plus sauvaige lieu et el mains hanté qui soit en nul lieu de mer habitable. Li lieus est si gastés et si desplaisans ke il n'i a ne tant ne quant de nule terriene soustenanche ne il n'i a de terre muable tant com uns poins porroit enclore, ançois est toute fine roche naïeve jusques es ondes de la mer. Et pour chou ke la roche siet en si sauvage lieu et en si perilleus, pour che est apielee des païsans la roche del Port Peril. (p. 187, § 304)

9 C'est à tel point que le personnage qui s'y trouve transporté, loin de pouvoir embrasser du regard le sud de l'Espagne et la cité de Cordoue comme l'annonçait plus haut le narrateur, ne voit «se les nues non deseur lui et environ soi ne vit se la mer non, fors ke tant seulement d'espasse com une petite roche tenoit: che fu li lieus ou il estoit " (p. 187, § 303). La dernière caractérisation topographique de l'île viendra d'ailleurs explicitement s'inscrire en faux contre la situation géographique effectuée par le narrateur en en reprenant le terme-clé («trespas ») pour le nier avec force :

Il vit [i.e. Mordrain] la roche gaste et hideuse comme chele qui en trop sauvage lieu estoit et destourné de tous trespas, se ensi non com aventure i amenoit aucune nef. (p. 206, § 332 $2^{10}$ )

10 C'est également un effet de brouillage des repères géographiques que l'on retrouve dans l'épisode consacré à l'île d'Hippocrate. Cet effet, moins spectaculaire que dans l'exemple précédent, se manifeste ici moins dans l'île proprement dite qu'à ses abords, tout se passant comme si les personnages, à mesure qu'ils s'en rapprochaient, perdaient tout moyen de se repérer dans l'espace. Le narrateur se montre d'abord très précis quant à leur situation géographique quelques jours avant l'embarquement : nous savons en effet qu'ils se trouvent à Tosqueham, en Égypte, lorsqu'ils apprennent que Nascien, qu'ils recherchent, se trouve en "mer de Grece». Se dirigeant vers la côte, ils font étape à Alexandrie, où ils perdent l'un des leurs. Le lieu de leur embarquement, néanmoins, manque déjà de précision. On ne nous donne en effet ni le nom du port, ni la distance exacte qui le sépare d'Alexandrie. Ce flou géographique ne tarde pas à augmenter : dans la nuit, le bateau, dont les voiles sont demeurées hissées, est emporté par un vent violent en haute mer, où il dérive durant trois jours avant de se fracasser contre l'île d'Hippocrate. Les personnages, relayés par le narrateur, ne manquent pas de souligner cette soudaine perte de repères : «il distrent bien entr'els, parmi tot le peril ou il estoient, qu'il ne pooit estre qu'il ne fussent trop loig de la terre d'Egypte : et sanz faille si estoient il, car la nef avoit toz dis alé aussi con se tuit li vent del monde la chaçassent. » (p. 344, § 538)

11 Dans ces deux exemples, le caractère isolé de l'île est loin de s'imposer d'emblée. Si l'île de Port Péril est située au carrefour d'itinéraires qui quadrillent le monde connu médiéval, reliant l'Est à l'Ouest et le Nord au Sud, celle d'Hippocrate, pour être moins passante, ne se situe pas non plus aux confins du monde, mais en Méditerranée, à trois jours de navigation de la côte égyptienne. Pourtant, l'une et l'autre finissent par brouiller les repères géographiques qui pourraient servir à les localiser précisément. L'espace insulaire est donc moins un espace qui se situerait hors de l'espace commun qu'un espace qui perturbe les repères de cet espace au point de les rendre caducs, soit qu'ils s'effacent subitement (Port Péril), soit qu'ils n'aient progressivement plus cours 
(île d'Hippocrate). Tout se passe comme si l'espace insulaire provoquait une faille dans l'espace-temps objectif, faille qui ferait échouer non seulement toute tentative de localisation géographique, mais aussi toute tentative de description topographique précise ou singulière. Pour la plupart strictement interchangeables, indistinctement " sauvages », " gastes » et " rocheuses ", les îles de l'Estoire s'apparentent au « désert ", ce lieu commun de la littérature d'édification qui n'a d'autre fonction que de permettre au diable de tenter l'homme et à Dieu de manifester sa toute-puissance.

L'espace insulaire de l'Estoire est donc un espace éminemment paradoxal, tout à la fois localisable et insituable : clairement et parfois soigneusement inséré dans un espace géographique référentiel, il en perturbe cependant systématiquement les repères. S'il permet d'autre part au récit de couvrir de l'espace et d'amorcer des itinéraires entre Orient et Occident, ces avancées spatiales, difficilement évaluables, sont réduites à néant à la fin de la seconde partie, où tous les personnages se retrouvent peu ou prou à leur point de départ. Mais il se trouve que ce paradoxe même répond au double projet du roman : écrire l'histoire d'une traversée d'Orient en Occident qui soit, en même temps, l'histoire d'un salut spirituel. Ou, pour le dire autrement, configurer un espace reconnaissable, assujetti à des repères géographiques aisément identifiables par le lecteur / auditeur, tout en laissant affleurer sous cet espace un espace autre, qui relève de repères psychiques et dessine une cartographie symbolique. Constitué par une longue tradition mythologique et hagiographique comme espace de l'ailleurs par excellence, l'espace insulaire, plus que tout autre, fournissait la scène idéale où manifester cet affleurement.

13 La plupart des îles de l'Estoire sont des lieux d'épreuves, comme le titre de la seconde partie : « les paours $»^{11}$ vient en témoigner avec éloquence. Pourtant, il est frappant de remarquer que le récit de ces épreuves ne constitue jamais le souci premier du narrateur lorsque les personnages parviennent dans l'espace insulaire. L'abordage dans l'île, l'évocation brève de sa situation et de sa topographie sont dans la grande majorité des cas immédiatement suivis d'un récit qui ne concerne apparemment en rien le ou les personnages qui s'y trouvent transportés, ce récit pouvant lui-même s'articuler sur des récits seconds. C'est à ces différents procédés de décrochage narratif que nous nous intéresserons désormais.

Récits enchâssés

14 Les récits secondaires qui s'enclenchent dans l'espace insulaire de l'Estoire frappent par leur nombre, leur longueur et surtout par leur diversité, tant dans les sujets abordés que dans les genres utilisés et les registres concernés : Histoire antique et biblique (île de Port Péril, île d'Hippocrate, île Tournoyante), mythologie gréco-romaine (île du roi de Sur, dite "isle au Jaiant", dont il est fait mention sur l'île Hippocrate), récit cosmogonique (île Tournoyante), anecdote de type évangélique (île Tournoyante), notice de Bestiaires (Port Péril), fabliau et exemplum (Hippocrate), histoire «à la manière arthurienne " (île du roi Orcauz), ou figurant déjà dans un roman arthurien (l'Arbre de Vie dans l'île Tournoyante). Ce bref relevé appelle deux observations simples. La première est que la plupart de ces récits seconds permettent à l'Estoire d'englober des strates temporelles plus anciennes que celles dans lesquelles la diégèse prend son origine. Les îles où s'effectuent ces récits secondaires fonctionnent ainsi comme autant de fausses fenêtres permettant de créer des effets ponctuels de profondeur temporelle, tout en maintenant l'histoire racontée dans le cadre d'une chronologie qui, pour être floue, n'en est pas moins limitée - en amont par la Passion, 
et en aval par l'évangélisation de la Grande-Bretagne. Le second élément significatif de ces récits secondaires, sensible à la seule énumération de leurs sujets, est qu'ils permettent à l'Estoire de prendre en charge, outre une partie de l'héritage de la Bible, plusieurs des principaux champs de savoir qu'avait investis la culture antique, en l'occurrence l'Histoire, la cosmologie et la zoologie. Considérer l'espace insulaire comme un vaste gisement culturel n'est pas une nouveauté. Comme l'a bien montré F. Lestringant, c'est dès la basse Antiquité que les îles de la Méditerranée, et en particulier les îles de l'Archipel égéen, constituent pour des géographes comme Pausanias ou Denys d'Alexandrie « tout à la fois la source de légendes et d'histoires [...] et le cadre taxinomique idéal où stocker une multitude d'informations venues d'un autre âge $\aleph^{12}$. Pour autant, il revient à l'Estoire d'avoir su employer les potentialités taxinomiques de cet espace à d'autres fins que celles de la description ou de l'inventaire. Car c'est bien une ambition essentiellement littéraire, et très spécifique à l'Estoire, que servent les récits d'îles qui se déploient ici : ancrer l'histoire du Graal dans l'épaisseur temporelle et l'efflorescence de la culture antique tout en la greffant étroitement sur la Bible. Témoin éclatant de cette ambition encyclopédique et totalisante est le double titre que le récit se donne : outre "Estoire del saint Graal ", titre que la critique a retenu, le roman s'intitule également, en effet, "Estoires de toutes les estoires » (p. 1, § 2), ou encore « Estoire des estoires » (p. 249, § 401).

À lire de près ces récits secondaires, et surtout à analyser la manière dont ils s'inscrivent dans le récit premier, il apparaît en outre que l'espace insulaire ne se donne pas seulement comme un espace privilégié où redéployer des traditions culturelles diverses et mettre en œuvre des formes narratives multiples. L'île représente aussi et peut-être surtout un espace poétique, c'est-à-dire un espace d'expérimentation littéraire où ces différents récits disparates trouvent à s'organiser, et où le risque de dispersion qu'ils impliquent peut être contenu. Essentiellement appréhendé comme hétérogène, toujours susceptible d'introduire dans le récit premier une parole interticielle capable de le faire dévier de sa «droite voie ${ }^{13}$, le récit second doit en effet être encadré. La clôture propre à l'espace insulaire fournit un moyen commode d'y parvenir. De fait, dans l'Estoire, les récits secondaires qui s'enclenchent dans un espace insulaire n'en dépassent jamais les limites: le narrateur revient toujours à son récit premier avant d'aborder une autre île, et aucun des récits secondaires n'est repris dans un autre espace insulaire (même si des effets d'échos peuvent se repérer d'un récit l'autre et d'une île l'autre, comme nous le verrons). De fait, la critique a depuis longtemps souligné (la plupart du temps pour le regretter) le caractère marginal des récits d'îles de l'Estoire, qui dans leur grande majorité pourraient être détachés du récit principal sans grand dommage ni pour celui-ci, ni pour eux-mêmes ${ }^{14}$. Comme le résume Michèle Szkilnik, "L'île est une possibilité d'excursus, de récit secondaire relié au récit principal mais qui, doté d'un début et d'une fin, conserve une certaine indépendance, pourrait être extrait de l'Estoire et fonctionner tout seul $\aleph^{15}$. Cette utilisation de l'espace insulaire à des fins d'excursus narratif serait cependant relativement banale si elle ne s'accompagnait du souci, remarquable par sa fréquence, de redoubler l'atout que représente en la matière la configuration naturelle de l'île par des dispositifs d'encadrement narratifs explicites et souvent très élaborés. Grâce à ces dispositifs, le décrochage suscité par l'émergence du récit second est à la fois mis en valeur et étroitement contenu par le récit premier. Nous ne retiendrons ici que les deux exemples les plus significatifs de ce procédé. 
16 Le premier de ces exemples concerne le récit relatant les démêlés de Foucaire et de Pompée, premier récit secondaire à s'enclencher dans une île. Le procédé d'encadrement est ici simple et efficace : il repose sur la reprise, à la fin du récit second, d'une séquence symétrique à celle qui avait marqué son début :

Séquence introductive du récit secondaire:

La roche est de tel hauteche com vous avés oï, et si est en tout le plus sauvaige lieu et el moins hanté qui soit en nul lieu de mer habitable. Li lieus est si gastés et si desplaisans ke il n'i a ne tant ne quant de nule terriene soustenanche [...]. (p. 187, § 304)

Récit secondaire : le combat de Pompée contre le pirate Foucaire (récit de type historique)

Séquence conclusive :

Atant se taist ore li contes de Pompee et si retorne au roi qui est en la roche [...]. Il esgarda entour lui : il ne vit nule riens fors mer et chiel, dont nule garisons li peüst venir ne nule soustenanche. La roche fu haute et en salvaige lieu; i tant d'abitacle com il i avoit, si estoit lais et hideus et escurs. (p. 197, § 319)

17 Comme on le voit, le cadrage du récit second est ici obtenu par divers effets d'échos: duplications pures et simples (le syntagme «sauvaige lieu», le substantif « soustenanche », le pronom indéfini « nul(e)», lui-même répété à l'intérieur de chaque séquence), polyptotes ( «hauteche»/ "haute»; « habitable»/«abitacle»), effets de redondance et de synonymie (« gastés et desplaisans » > « lais et hideus et escurs »; « el moins hanté qui soit en nul lieu de mer habitable » > « il ne vit nule riens fors mer et chiel »). On soulignera également la structure chiasmique qui verrouille l'encadrement $\mathrm{du}$ récit second: les deux syntagmes explicitement repris dans la séquence de clôture ("soustenanche» et «salvaige lieu») sont inversés par rapport à la séquence d'ouverture.

C'est dans l'épisode de l'île d'Hippocrate que l'on trouve la version la plus élaborée du procédé d'encadrement du récit second. Ce récit s'enclenche à l'occasion de la découverte des ruines d'un palais autrefois construit sur l'île. Ces ruines abritent encore une tombe qui porte l'épitaphe suivante: «Ci gist Ypocras, li soverains des fisiciens, qui par l'engin de sa feme reçut mort et fu ci aportez par Antoine, lo roi de Persse » (p. 348, § 544). On peut d'abord s'arrêter sur les indications qui précèdent la découverte de cette épitaphe par les personnages. Elles mettent toutes en lumière, en effet, des configurations spatiales encadrantes ou emboîtantes : en pénétrant dans le palais, les personnages passent d'abord par une "huisserie de marbre vermeil ", vestige de l'entrée, puis, avançant entre des piliers de marbre jusqu'au bout du palais, aperçoivent, sous un arc voûté, un lit qui supporte la tombe où se trouve gravée l'épitaphe d'Hippocrate. Si c'est l'énoncé de cette épitaphe qui enclenche véritablement le récit secondaire qui nous intéresse, il faut souligner que ce récit est encore précédé d'une dernière intervention du narrateur, qui commente le comportement des personnages découvrant l'épitaphe :

Qant il voient les letres, si les conoissent molt bien et em parolent assez et dient que d'Ypocras avoient il oï assez parler mainte foiz. Il regardent la meson de chief en chief, si voient trop de beles choses qui totes estoient tornees a naient et dechaoites et dient que iluec ot jadis molt riche manoir et mout bel [...]. (p. 348, § 544)

Commentaire intéressant en ce qu'il insiste sur l'écho - ou plutôt sur la cascade d'échos - que suscite la lecture de l'épitaphe d'Hippocrate. Les personnages lui ménagent d'abord un écho en en glosant longuement le texte ( « em parolent assez »), puis cette première glose se fait elle-même l'écho de paroles anciennes (« dient que d'Ypocras avoient il oï assez parler »). Enfin, à un autre niveau d'analyse, un troisième 
effet d'écho se fait entendre dans le discours du narrateur lorsqu'il reprend en chiasme les mêmes termes autour du verbe "dire » (" parolent assez » / " assez parler »), tout se passant alors comme si les échos emboités des paroles fictionnelles avaient contaminé la parole du narrateur... vouée elle-même, le cas échéant, à être redoublée par celle du conteur.

Le récit secondaire proprement dit débute en ces termes :

Mais or laisse li contes ci endroit a parler d'els [i.e. les personnages qui commentent l'épitaphe] por conter la verité de cele meson que Ypocras fist fere por son manoir : et comment ele fu estoree et atornee si richement et par qel maniere, et qui cil fu qui l'estora, et coment cele meson, qui jadis ot esté si riche et si bele, fu einsint dechaoite. (p. 349, § 545)

et se termine sur ce paragraphe :

Par tel maniere come je vos ai devisee fu la meson establie premierement si riche et si bele come je vos ai conté et après fu desertee si malement. Si s'en test ore atant li contes, quar assez longuement en a parlé et retorne en une autre voie. (p. 369, § 577).

Le dispositif d'encadrement repose sur les procédés déjà repérés de la duplication («par qel maniere» / «par tel maniere»; «si riche et si bele ») et de la synonymie (« estoree »/« establie»; « jadis »/« premierement»; « dechaoite»/« desertee »), auxquels viennent ici s'ajouter deux commentaires du narrateur qui se font écho. L'un est proleptique ( «... por conter la verité de cele meson... »), et l'autre analeptique («par tel maniere come je vos ai devisee »). Pourtant très explicite, cette structure d'encadrement se voit redoublée par une autre : avant de retrouver le récit premier, le narrateur revient encore une fois sur la découverte de la tombe d'Hippocrate et rappelle de nouveau, en un ultime effet d'écho, les commentaires qu'elle a suscités :

Ci endroit dit li contes que, qant li dui mesage et la damoisele qui avec els estoit orent grant piece regardee la maison et la tombe Ypocras et il conurent par les letres qui desus la tumbe estoient qe li sages philosophes Ypocras avoit en tel maniere mort receüe par l'engin de sa feme, si en commencent entr'els a parler et distrent que molt avoit esté cist damages granz, et deable chose et mout dotable avoit en feme, car encontre son enging ne puet sens d'ome durer. (p. 370, § 578)

Il faut donc parler ici d'une double structure d'encadrement du récit second, ou, plus précisément, de la présence de deux cadres emboités l'un dans l'autre. Le premier cadre - le plus large - est constitué par deux séquences consacrées à la découverte de la tombe d'Hippocrate et aux discours qui l'ont accompagnée. Le second, contenu par le premier, est formé par les deux paragraphes cités plus haut, qui annoncent respectivement le début du récit secondaire et sa fin. Il faudrait encore souligner que ce dispositif d'enchâssement sophistiqué, où les structures d'emboîtement se dupliquent et les effets échos se démultiplient, entre en résonance avec une réflexion complexe sur le temps qui passe, la mémoire historique et affective, le vestige et la trace, qui mériterait une étude à elle seule.

Les exemples que nous venons d'analyser tendent à montrer que l'espace insulaire de l'Estoire est un espace propre non seulement à susciter le surgissement de récits divers, dans le sens que le Moyen Âge accorde à cet adjectif (à la fois variés et disparates), mais aussi à structurer leur organisation selon des dispositifs qui frappent par leur netteté et parfois leur complexité. On pourrait cependant montrer que l'obsession de la contention des récits secondaires - obsession qui se lit notamment dans la redondance des dispositifs d'encadrement et d'emboîtement - est contrebalancée dans la plupart des cas par l'existence de liens (narratifs, structurels ou thématiques) qui se nouent 
entre ces récits et le récit principal, mais aussi entre ces récits eux-mêmes. Si comme nous l'avons dit un récit enchâssé ne dépasse jamais les limites de l'espace insulaire dans lequel il se déploie, il peut toutefois résonner dans des récits enchâssés qui s'enclenchent sur d'autres îles. Ainsi, pour nous en tenir uniquement aux exemples que nous avons convoqués, l'histoire qui met en scène Foucaire et Pompée dans l'île de Port Péril entre en résonance avec celle d'Hippocrate: elles recèlent toutes deux des vestiges qui font affleurer des fragments d'Histoire antique, et posent toutes deux la question de la continuité de cette Histoire dans un temps et un espace orientés par le christianisme. On pourrait ainsi mettre en évidence un certain nombre de lignes narratives secondaires se dessinant, plus ou moins nettement, entre les récits enchâssés qui se développent dans l'espace insulaire de l'Estoire. Certaines de ces lignes secondaires croisent les grands axes du récit principal (c'est le cas de la réflexion sur la stratification des époques et des cultures qui se fait jour dans les îles de Port Péril et d'Hippocrate), quand d'autres se poursuivent parallèlement à lui (telle, par exemple, l'exploration de diverses histoires de couples, qui illustrent au fil des îles - île d'Hippocrate, du Géant, du roi Orcauz - les différentes manières dont les femmes peuvent faire basculer le destin de héros masculins). La cartographie précise de ces lignes secondaires et de leurs transversales reste à établir, si tant est qu'une telle entreprise soit réalisable. C'est donc sous la forme du conditionnel que nous développerons l'hypothèse suivante: plus qu'un espace de contention d'une parole perçue comme digressive, l'espace insulaire serait utilisé comme un moyen inédit de faire coexister simultanément plusieurs lignes narratives. Pour ce faire, le roman en prose usait jusque-là du procédé de l'entrelacement. L'Estoire inaugurerait celui de l'écriture en réseau. ${ }^{16}$

Récits d'origine

Si l'on excepte l'île de l'épée brisée et l'île du roi Orcauz, les récits secondaires qui surviennent dans l'espace insulaire de l'Estoire sont tous des récits rétrospectifs. L'abordage dans l'île suscite presque immédiatement un « retour amont » qui relie le « jadis » au présent de l'histoire racontée et qui peut susciter une réflexion sur ce lien. Ces récits rétrospectifs confèrent une dimension archéologique à l'espace insulaire, et ceci non seulement parce qu'ils concernent pour la plupart l'Antiquité, mais aussi parce qu'ils s'organisent autour de la découverte de vestiges et/ou de recherches toponymiques. Le décrochage narratif concernant Foucaire se légitime d'abord par une enquête sur le nom de « Port Péril » que les indigènes donnent à l'île. Il est également justifié, un peu plus loin dans le récit, par la découverte que fait Nascien de la grotte où les naufrageurs se cachaient et où Pompée aurait livré l'assaut final. L'histoire d'Hippocrate, nous l'avons vu, est racontée à la suite de la découverte des vestiges du palais naguère construit par le personnage et de l'épitaphe gravée sur sa tombe. Le récit cosmologique qui s'enclenche dans l'île Tournoyante a également pour fonction première d'éclaircir les raisons pour lesquelles les « paysans » ont donné ce nom à l'île. Enfin, la brève notation sur le combat ayant opposé Hercule et un géant sur l'île du roi et de la reine de Sur n'a d'autre raison d'être que de légitimer en droit le nom de l'île, $l^{\prime}$ « île au Jaiant». Si tous les vestiges pseudo-historiques du roman ne se situent pas dans des îles, et si de nombreux toponymes dont le narrateur établit l'origine ne sont pas insulaires, on soulignera cependant que les îles de l'Estoire se caractérisent par le lien privilégié qu'elles entretiennent avec le passé, et que ce lien, dans tous les cas, se manifeste par la permanence d'un témoin de ce passé dans le présent de la diégèse. L'écriture romanesque exploite ici une caractéristique de l'espace insulaire 
historiquement et scientifiquement attestée: constituer un conservatoire naturel d'édifices, de noms ou d'espèces peu représentés ou disparus ailleurs ${ }^{17}$. Cette caractéristique de l'espace insulaire "réel» est exploitée par la fiction romanesque pour s'enraciner dans un passé susceptible de lui apporter une garantie d'authenticité historique. Mais elle sert aussi et surtout de prétexte au déploiement systématique de récits rétrospectifs de type explicatif qui fournissent une légitimation supplémentaire au titre que le récit se donne à lui-même, «Estoire des estoires ». Nous l'avions lu plus haut comme l'affirmation de l'ambition encyclopédique de l'œuvre. On peut aussi l'interpréter, à la lumière de ces récits, comme l'affirmation (non exclusive de la première) d'une ambition archéologique, au sens foucaldien du terme : si l'Estoire del saint Graal peut être dite " estoire des estoires ", c'est aussi parce qu'elle vise à mettre au jour, et ceci par des récits (des « estoires»), l'origine de tous les récits (de toutes les " estoires »).

Or il s'avère que tous les récits qui se déploient dans le cadre de l'espace insulaire de l'Estoire sont des récits qui rapportent quelque chose d'une origine. Cette unanimité est remarquable, d'autant qu'elle rassemble aussi les très rares récits insulaires qui ne soient pas explicitement étiologiques et/ou qui ne soient pas seconds par rapport au récit principal. Que les récits rétrospectifs que nous avons jusque-là étudiés puissent être considérés comme des récits d'origine tient évidemment à leur statut étiologique, mais aussi au fait que ce statut se manifeste la plupart du temps sous la forme d'une enquête étymologique. La recherche de l'étymologie d'un nom, et surtout d'un nom propre, est en effet pour les penseurs médiévaux la voie d'accès la plus sûre à son origine première et à son sens véritable, "origine » et "vérité " étant en la matière synonymes. Mais si l'on peut dire de tous les récits insulaires de l'Estoire qu'ils sont des récits d'origine, c'est avant tout parce qu'ils ont pour fonction de conférer au roman le statut qu'il revendique: celui d'un texte orignaire, qui non seulement fixe les commencements du cycle du Lancelot-Graal, mais en assure aussi la fondation et la cohérence. Ainsi des récits secondaires qui rapportent des histoires se déroulant dans l'Antiquité. S'ils permettent au narrateur, comme nous l'avons vu, de repousser les limites temporelles de son récit, on pourrait également montrer qu'ils lui offrent surtout, ce faisant, l'occasion d'insérer dans la matière historico-biblique sa propre matière fictionnelle. Une fois étroitement imbriquée par l'artifice de l'écriture à la Bible et/ou à l'histoire antique, où elle n'avait naturellement à l'origine aucune part, la fiction romanesque peut prétendre à un statut aussi fondateur, sinon plus, que ces deux traditions textuelles. Nous ne nous arrêterons cependant pas sur ce type de récits, tant pour en avoir étudié ailleurs un exemple paradigmatique ${ }^{18}$, que pour nous donner le temps d'évoquer deux récits insulaires sur lesquels nous ne nous sommes pas encore arrêtée, et qui sont à présent susceptibles d'ouvrir de nouvelles perspectives à l'analyse. Il s'agit des récits de l'île du roi Orcauz et de l'île de l'épée brisée, où le statut originaire de l'Estoire s'établit moins par rapport à la tradition historique ou biblique que par rapport à la fiction du Graal et à la matière arthurienne de manière générale.

L'île du roi Orcauz est la dernière île dont le récit fasse mention; elle ne sert de cadre à aucun récit secondaire, qu'il soit rétrospectif ou non. Pourtant, l'histoire qui s'y déroule n'est pas sans intérêt pour le sujet qui nous occupe ici. Elle peut en effet être lue dans son ensemble comme une sorte de prologue propre à initier le lecteur / auditeur aux romans d'amour et de chevalerie, et plus particulièrement aux romans arthuriens, tant elle en concentre en quelques pages les lieux communs les plus manifestes et les ficelles narratives les plus usées (princesse amoureuse d'un inconnu 
qu'elle sauve de la mort, prouesses chevaleresques à répétition, incognito et révélation d'identité spectaculaires, etc.). Cette initiation en accéléré aux motifs de la littérature arthurienne se termine sur le baptême du roi Orcauz. Le roi change de nom à cette occasion, mais il a la bonne idée de léguer son ancien nom à son château, qui devient donc le château d'Orcanie. Or ce toponyme est bien connu de la matière arthurienne, puisqu'il désigne le royaume de Loth, père de Gauvain et de ses trois frères. Avant de quitter l'île, le narrateur établira d'ailleurs la généalogie des rois d'Orcanie depuis Orcauz jusqu'aux quatre fils du roi Loth, manière de confirmer, s'il en était besoin, le statut tout à la fois introductif et programmatique de ce dernier récit insulaire. L'île de l'épée brisée, quant à elle, n'a à première vue d'autre fonction que d'ajouter un épisode supplémentaire aux aventures de Nascien. À peine arrivés dans cette île, ce personnage et son fils Célidoine sont pris à parti par un géant qui leur reproche d'avoir abordé sans sa permission. Pris de court, Nascien prend dans la Nef de Salomon l'épée du roi David, laquelle se brise entre ses mains, signe qu'il n'était pas digne de l'utiliser. Fort heureusement, il ne tarde pas à en trouver une autre, grâce à laquelle il finit par avoir raison du géant. D'un intérêt narratif limité, apparemment dépourvue de tout lien avec l'économie du récit principal, cette histoire prend en revanche tout son sens lorsqu'on la met en perspective avec la suite du cycle, et plus particulièrement avec son avantdernier volet, la Queste del saint Graal. La brisure de l'épée y est en effet mentionnée comme ayant déjà eu lieu dans un passé lointain, mais ne fait l'objet d'aucun récit. En donnant les détails de cette aventure, et surtout en la rapportant au présent, c'est-àdire en l'insérant dans la chronologie de l'histoire qu'elle raconte, l'Estoire s'affirme sans doute possible, bien que rétrospectivement, comme antérieure à la Queste. De ce point de vue, c'est surtout l'ensemble du récit consacré à la Nef de Salomon, à son histoire et à son contenu, qui peut être interprété en ce sens. Ce récit, qui s'insère dans l'épisode consacré à l'île Tournoyante, reprend en effet presque terme à terme un récit déjà présent dans la Queste del saint Graal. On voit mal ce qui pourrait légitimer cette longue reprise sinon la volonté d'imposer l'œuvre comme le premier volet du cycle : puisque la diégèse de l'Estoire del saint Graal est chronologiquement antérieure à celle de la Queste del saint Graal, c'est la Queste qui paraîtra reprendre l'Estoire et non l'inverse. Mais il y a mieux. Car toute l'entreprise de la fabrication de la Nef ne prend sens qu'en vue de la venue de Galaad, le héros de la Queste, celui qui mettra un terme définitif aux aventures du Graal. En rapportant l'histoire de cette fabrication et en annonçant la venue de Galaad, l'Estoire del saint Graal ne se situe pas seulement au commencement du Lancelot-Graal. Elle en constitue aussi le récit fondateur au sens fort, un récit capable d'embrasser la fin du cycle et d'en assurer ainsi, a posteriori, la cohérence d'ensemble.

Que l'espace insulaire de l'Estoire soit davantage un espace symbolique qu'un espace référentiel ne saurait surprendre dans un récit qui affirme la prééminence du paradigme interprétatif chrétien. Ce qui constitue en revanche une spécificité remarquable du récit est que l'île y est systématiquement traitée comme un espace poétique au sens propre du terme, grâce auquel le roman parvient à remplir le lourd et paradoxal « cahier des charges » que lui impose sa situation au sein du Lancelot-Graal. La grande habileté du narrateur est d'avoir en l'occurrence su transformer en possibilités narratives des caractéristiques traditionnellement associées à l'île, qu'on la considère comme un espace « réel » (géographiquement et historiquement situable) et/ou comme un espace imaginaire. Partant de la représentation géographique de l'île comme espace clos et (relativement) isolé, le narrateur a ainsi fait de l'espace insulaire un espace où expérimenter de multiples possibles narratifs sans perturber le déroulement du récit 
principal. De même, le rôle de conservatoire joué par l'espace insulaire, rôle historiquement attesté, est ici utilisé pour susciter l'enclenchement de divers récits rétrospectifs propres à enraciner le roman dans l'épaisseur des strates temporelles préchrétiennes et à en faire l'héritier du savoir polymorphe de l'Antiquité. Mais c'est plus largement le fantasme qui associe l'espace insulaire à un espace originel qui semble mobilisé dans le cas des récits d'îles de l'Estoire : tous, en effet, ressortissent à un récit originaire. En posant ce rapport de convenance ou plutôt de nécessité entre l'île et le récit d'origine, le narrateur œuvre là encore à consolider la position de l'Estoire au sein du Lancelot-Graal. Car qu'ils soient de type étiologique ou de type programmatique, qu'il s'agisse de mettre au jour l'histoire de vestiges architecturaux, l'origine d'un toponyme ou bien de mettre en place l'avenir et la fin de la trajectoire romanesque du Graal, ces récits tout à la fois construisent et confirment le statut fondateur que le roman doit assumer au sein du cycle.

\section{NOTES}

1. Le Perlesvaus, la Queste del saint Graal se terminent sur de telles navigations, et l'on sait l'importance de l'Irlande dans les récits tristaniens et de l'île d'Avalon dans la légende arthurienne - Avalon constituant d'ailleurs pour le Joseph de Robert de Boron le lieu d'arrivée du Graal en Occident.

2. La question de la date de composition du roman fait encore débat. La grande majorité des critiques plaident cependant pour l'hypothèse de la postériorité de l'Estoire sur l'ensemble du cycle, et considèrent qu'elle a dû être composée vers 1230-1235. Nous partageons cette thèse, dont on pourra se faire une idée en consultant les travaux suivants : A. Pauphilet, Études sur la Queste del Saint Graal, Paris, 1921, J. Frappier, Étude sur la Mort le Roi Artu, Genève, 1938 et, plus beaucoup plus récemment, M. Szkilnik, L'Archipel du Graal, Genève, 1991, et «L'Estoire del saint Graal : réécrire la Queste ", Arturus Rex : volumen II, Acta Conventus Lovaniensis 1987, Leuven, 1991, W. Van Hoecke, G. Tournoy et W. Verbeke éd., p. 294-305.

3. Nous nous intéresserons donc essentiellement à la spécificité des liens qui se nouent entre d'une part l'espace insulaire et d'autre part l'élaboration et le statut du récit. Le rôle de métaphore ou de modèle (au sens de modèle mathématique) que peut jouer la figure de l'île et surtout de l'archipel dans l'appréhension de la structure du récit ou de l'organisation du cycle ne nous retiendra que de manière secondaire. Sur cette dernière question, on se réfèrera à M. Szkilnik, L'Archipel du Graal, op. cit. et F. Lestringant, Le livre des îles. Atlas et récits insulaires de la Genèse à Jules Verne, Genève, 2002.

4. La Grande-Bretagne, lieu de référence des aventures arthuriennes, n'est pas traitée par le récit comme un espace insulaire. Nous ne la prenons donc pas en compte dans notre étude.

5. Cf. notamment cette explicitation du personnage de Josephé : « ... la mer, ce est li mondes... », p. 415, § 653.

6. P. Zumthor, La mesure du monde, Paris, 1993, p. 51. 
7. C'est cette orientation spirituelle qui empêcherait l'Estoire de compter parmi les récits que F. Lestringant appelle des « insulaires-récits », ou " récits en archipel », dont l'une des caractéristiques est précisément de se déployer librement, hors de tout principe organisateur repérable (Le livre des îles, op. cit., p. 222 sq.).

8. Au sujet de l'île de Port Péril (p. 206, § 332), de l'île Tournoyante (p. 249, § 400) et de l'île d'Hippocrate (p. 345, § 540).

9. Notre édition de référence est celle de J.-P. Ponceau, Paris, 1997.

10. Dans cette citation comme dans les suivantes, c'est nous qui soulignons.

11. Fait assez exceptionnel pour être souligné, ce sous-titre figure dans le prologue même de l'œuvre.

12. Op. cit., p. 57.

13. On se reportera par exemple à cette intervention du narrateur en conclusion de l'un de ces récits seconds : "Or est drois que l'estoire soit ramenee a la droite voie dont li contes s'est auques departis pour parler de ches choses que il a amenteües, qui se fierent entre les paroles de l'estoire et si n'en sont mie. Mais la droite voie repaire a l'isle dont li contes a devisé et le lonc et le lé (...).» (p. 259, § 417)

14. Les reproches se sont surtout concentrés sur l'histoire d'Hippocrate, unanimement considérée comme l'exemple achevé du caractère composite du roman. Sur cette question, voir M. Séguy, « Hippocrate victime des images. À propos d'un épisode déconcertant de l'Estoire del saint Graal », Romania, t. 119, 2001, p. 440-464.

15. L'archipel du Graal, op. cit., p. 138.

16. Nous employons ce terme non dans le sens que F. Lestringant lui accorde en reprenant la figure du rhizome de G. Deleuze, mais dans le sens où l'on peut parler d'un réseau ferroviaire : si compliqué que puisse paraître le croisement des voies, on y repère toujours une structure concertée et hiérarchique, ce qui n'est pas le cas de l'organisation en rhizome.

17. On sait par exemple que les îles et les côtes orientales de la mer Égée ont connu, dès l'Antiquité, de longues périodes de désertification. Ce n'est qu'à partir de la seconde moitié du ix ${ }^{\mathrm{e}}$ siècle que s'est amorcé un mouvement de repeuplement de ces îles, dont le paysage était donc marqué par les ruines. Sur ce sujet, voir notamment S. Vilatte, L'insularité dans la pensée grecque, Paris, 1991.

18. Voir « Vestiges historiques et mémoire romanesque dans l'Estoire del saint Graal », Bien dire et bien aprandre « Histoire et roman », $\mathrm{n}^{\circ} 22,2004$.

\section{RÉSUMÉS}

L'Estoire del saint Graal constitue le premier volet du cycle romanesque du Lancelot-Graal. En tant que tel, ce récit doit fixer les débuts de la trajectoire du Graal, ménager des liens entre l'Antiquité païenne et les temps arthuriens, mais aussi assurer autant que faire se peut la cohésion d'un immense ensemble constitué de massifs narratifs hétérogènes. Ce difficile " cahier des charges " est compliqué du fait que l'Estoire a certainement été composée après l'ensemble du cycle dont elle doit écrire les origines et inventer la cohérence. L'article teste l'hypothèse selon laquelle l'espace insulaire, espace largement privilégié par le roman, offre un certain nombre de solutions 
narratives permettant de réussir cette entreprise ambitieuse et paradoxale. Dans cette perspective, l'espace insulaire apparaît moins comme un espace référentiel ou symbolique que comme un espace poétique, c'est-à-dire comme un lieu d'expérimentation littéraire où le récit trouve à se construire selon les contraintes particulières qui lui sont imposées par sa situation et sa fonction au sein du cycle.

Tales of the Isles. Insular Setting and the Poetics of Narrative in the Estoire del saint Graal. L'Estoire del saint Graal constitutes the first chapter of the romantic cycle Lancelot-Graal. As such this tale must not only set out the beginnings of the trajectory of the Grail and show the links between pagan antiquity and the Arthurian epoch, but also ensure as far as possible the cohesion of the enormous whole made up of a mass of heterogeneous narratives. This difficult schedule is complicated by the fact that the Estoire was certainly composed after the cycle whose origins and coherence it had to describe. This article tests the hypothesis whereby the insular setting, a setting particularly privileged by the story, offers a certain number of narrative solutions that favour the success of this ambitious and paradoxical enterprise. In this perspective the insular setting appears less as a referential or symbolic setting than as a poetic one, that is to say as a region of literary experimentation where the narrative is constructed according to the particular constraints that are imposed on it by its place and function within the cycle.

\section{INDEX}

Keywords : Estoire del saint Graal, insular setting, poetics of narrative, prose novel, narrative cycle

Mots-clés : espace insulaire, poétique du récit, roman en prose, cycle

\section{AUTEUR \\ MIREILLE SÉGUY}

Université Paris VIII Vincennes-Saint-Denis, 2, rue de la Liberté, F-93526 Saint-Denis Cedex 